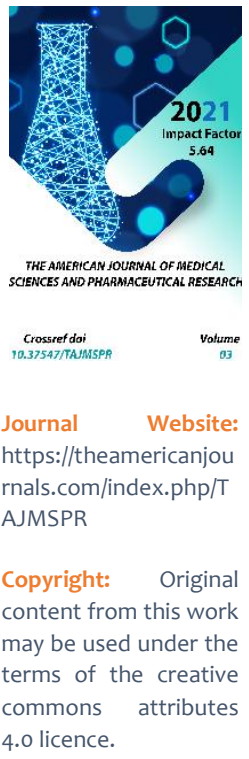

\title{
The Importance Of Lyophilization Of Microorganisms And Biologicals
}

Okiev S.S.

Applicant (PhD) State Scientific Center For Quality Control And Circulation Of Veterinary Medicines And Feed Additives, Uzbekistan

\section{ABSTRACT}

This article provides arguments about the need for scientific research aimed at maintaining the stability of biological properties during long-term storage, production, reference, epizootic strains of microorganisms through the development and optimization of lyophilization parameters.

\section{KEYWORDS}

Protective environment, storage methods, collection strain, lyophilization, survival, cryoprotectant, microorganism.

\section{INTRODUCTION}

Currently, lyophilization, or freeze drying, is one of the most modern methods of preserving biological preparations (vaccines, microorganisms, serums, and others), which allows maintaining the basic physical and biological properties during storage for several years.

The growth in the number of animals and birds in Uzbekistan is forcing state veterinary institutions to increase the number of vaccines and diagnostics used to maintain persistent epizootic and epidemiological well-being in the country.

One of the main problems hindering the development of livestock and poultry farming in the country is the lack of industrial production of immunobiological preparations. Since the timely implementation of planned antiepizootic measures depends on the availability of a sufficient amount of highquality vaccines, diagnosticums, and other 
means of preventing infectious diseases of agricultural animals.

In Uzbekistan, the production of high-quality immunobiological preparations directly depends on the availability of reference, production and control (reference) strains of microorganisms, as well as the conditions for their preservation and maintenance, which is one of the main tasks of the development of biotechnological production in the veterinary field. Therefore, in our country, as in any other priority task facing veterinary science, is the creation of its own collection of standards of industrial strains of microorganisms, which, in fact, are technologies for production.

These collections in many countries are created by selection and careful study of the biological properties of microorganisms, the stability of their inherent genetic traits (markers), persistence in the body of animals, the interaction between macro and microorganisms.

An important link in the production of immunobiological preparations is the method of lyophilization or freeze drying, in which it is possible to achieve maximum preservation of the basic properties of the product. Therefore, in the passport of a production strain of a microorganism and in other regulatory documents, methods of storing and maintaining production strains and other drugs are mandatory.

Currently, the main method of maintaining live production strains and reference cultures is the method of subculture and storage on nutrient media. At the same time, the change in virulence, biochemical and other properties is well known during prolonged passages on nutrient media and on which the effectiveness of manufactured vaccines and diagnosticums directly depends.

For the first time, the lyophilization method was substantiated and patented in the USSR by engineer G.I. Lappa-Starzhenetsky back in 1921. But due to insufficiently developed vacuum and refrigeration technology, it did not spread until the 40s. Starting from the 50 s of the last century and up to the present time, employees of the All-Union State Scientific and Control Institute of Veterinary Medicines, together with specialists from the biological industry, have developed and introduced into industrial production more than 30 dry biological products. (S.G. Kolesov, Yu.P. Chernetsky, 1981). The main purpose of lyophilization was to ensure the implementation of therapeutic and prophylactic measures in the country with dry drugs. In addition, the introduction of laboratory methods of freeze drying served for the conservation and standardization of cultures of bacteria, viruses, sera and the production of small batches of biological products.

It should be noted here that the process of lyophilization of microorganisms and biological products has a number of fundamental features in comparison with the drying of other materials. First of all, this applies to drying objects - microbial and viral suspensions, biological dispersed systems, the presence of moisture in which reaches $96 \%$. Strains of microorganisms and biological products, unlike other materials, are frozen and dried in glass vessels (ampoules, vials), which impose certain restrictions on heat and mass transfer during the drying process, the development of a continuous lyophilization process, and compliance with sterility. Freeze drying of biological products in comparison with other materials is deeper - the moisture content of 
the dried products should not exceed 3\%, with a drying duration of 24-72 hours. Drying is carried out at low pressures (0.1-0.05 millimeter mercury). All dried preparations are subjected, in addition to physical and chemical control, to mandatory biological verification.

The basic principles of lyophilization as applied to microorganisms and biological products are directly related to microbiology, virology, biochemistry, cryobiology and other biological sciences.

The entire lyophilization process includes two main stages:

- freezing, in which microorganisms and biological products in ampoules and vials are placed in cassettes and placed in lowtemperature chambers operating at temperatures from minus 40 to minus $600 \mathrm{C}$. In this case, freezing is carried out gradually, i.e. at atmospheric pressure up to minus $250 \mathrm{C}$, then within 15 minutes up to minus $400 \mathrm{C}$. This freezing method prevents material from contamination and shortens the freezing time by $30-35 \%$ compared to conventional methods. It should be noted that the process of freezing microorganisms and biological products includes four temperature regimes: the first from the initial temperature to oo $\mathrm{C}$, the second - from 00 to minus $50 \mathrm{C}$, followed by a "temperature reverse" to cryoscopic temperature, the third period is the phase transformation of water into ice, the fourth period is the hardening of a mixture of ice and liquid. The fourth stage is decisive, since complete solidification occurs after the state of the eutectic and the eutectic temperature (Tev), which is necessary to determine the upper and lower temperature limits and determine the eutectic zone. So, according to literary sources, it is in the range of 10-150 C.
The mechanism of action of low temperatures on strains of microorganisms, biological products and other biological objects is the main issue of cryobiology, but this issue has not yet been fully resolved. Today it has been proven that low temperatures mainly act on cell membranes, while their effect on bacteria, viruses, fungi, and tank preparations is different and has its own parameters (Mazur, 1974; Merimen 1976; Pushkar, 1978).

The second main stage of lyophilization is freeze drying. In this case, the frozen product is quickly transferred to the drying chamber, sealed, and a vacuum pump is turned on to remove moisture vapor from the frozen material in a vacuum. The freeze drying period is also divided into two periods. The first period is the drying time at subzero temperatures, the second period is the drying time at positive temperatures. Both periods are aimed at removing free and bound moisture.

All manufacturers of immunobiological preparations in the world have sublimation devices and their types are diverse. And although the basic principles of lyophilization are known, for each industrial strain and reference cultures of microorganisms, biological products, these parameters and conditions for lyophilization are developed, optimized separately and have their own characteristics.

In the literature, there is a lot of information that the loss of moisture leads to a slowdown in the vital activity of the microbial cell, which entails biochemical changes in many types of bacteria, while most microbes quickly lose their viability. At the same time, a very important fact of the influence on the survival of microbes of residual moisture, for example, in 
E. coli, staphylococcus, pseudomonas, was noted. (Okhapkina V.Yu., Shabalin B.A., 2009).

The most resistant microbes to drying are streptococci, especially pyogenic, and staphylococci, their survival rate ranges from 72 to $100 \%$. The group of bacteria with an average degree of resistance includes Salmonella, Shigella, Brucella, the survival rate of which ranges from 10 to $30 \%$. Storage depends on a number of factors: the age of the culture, the cultivation conditions, the composition of the nutrient medium for growing, the mode and method of lyophilization, the stabilizing medium, the amount of residual moisture, storage conditions, etc. (Arkadieva Z.A., 1983). When a 7-9-day culture of tuberculosis bacteria of the $B C G$ vaccine strain was lyophilized, the survival rate of microbes was $68,0-87,5 \%$, in a vaccine from a 10-day culture $-33,0 \%$, and from a 17-day culture - only 3,2\%. Schultz and Ritz found that E. coli cells are most resistant to lyophilization in the logarithmic growth phase.

The addition of $2 \%$ glycerol to the medium has a beneficial effect on the lyophilization of microbes. There is evidence of a correlation between the intensity of the formation of free radicals and the death of microorganisms (Heckly R.I., Dimnick R.L., Windle I.I., 1963). There are indications that up to $26-45 \%$ of ribonucleic acid is destroyed in the cell during drying (Timoshin A.A., Rappoport A.I., Beker M.E. 1990). The storage atmosphere has a marked effect on the viability of the dried bacteria. S.G. Kolesov showed that the presence of air in ampoules with dried cultures led to their complete death after 15 months of storage, while similar cultures stored under vacuum were stored for up to 4-6 years. Dried museum strains must be stored at subzero temperatures, which allows them to be "refreshed" no more than once every 5-10 years. Among the complex protective media containing serum, it should be noted the medium called "Mist. desiccans" proposed by Fry and Greaves. In addition to serum, 5, 10, 25\% solutions of individual fractions of serum proteins and blood proteins are successfully used. Media, in which gelatin and agar-agar are introduced as a colloid, are especially widely used in the production of dry live vaccines, since they are devoid of antigenic properties. In our country, the Faibich environment was previously used, which exists in two modifications: 1 . Sucrose $10 \%$, gelatin $1-1,5 \%$, agar-agar $0,05-0,2 \% ; 2$. Sucrose $10 \%$, gelatin 1 1,5\%. Subsequently, M.M. Faibich recommended adding antioxidants to these media: $1 \%$ ascorbic acid or $1 \%$ thiourea. These additives increase the stabilizing effect of the medium and allow the dried cultures to be stored without creating a vacuum. Potassium iodide and ammonium molybdenum can also be used as antioxidants.

Thus, long-term world science and practice confirms the need for scientific research on the lyophilization of microorganisms as a way of long-term maintenance of production strains and reference cultures with unchanged properties, which is important in bioproduction.

The purpose of our research is to study and optimize the parameters and conditions of lyophilization in relation to those sublimation installations that are available at the Research Institute of Veterinary Medicine and the State Scientific Center for Quality Control and Circulation of Veterinary Medicines and Feed Additives.

The aim of our research is to study various protective media (stabilizers) based on: blood 
serum of animals, gelatin, gelatose, peptone, sucrose, glycerin and others, and their combination. At the same time, the authenticity of production, reference, epizootic strains of microorganisms will be studied by studying biochemical, biological and other properties of microorganisms and a comparative analysis of the quality of biological products and collection strains obtained with their help.

\section{MATERIALS AND METHODS}

As test cultures, we used the standards of industrial strains Brucella abortus $19 \mathrm{UZ}$ variant, 104M UZ variant, Brucella melitensis Rev-1 UZ variant intended for the production of experimental series of anti-brucellosis vaccines. The study of biological properties was carried out according to the methods recommended by the Food and Agriculture Organization / World Health Organization for working with Brucella cultures. All work with the bacterial culture was carried out under sterile conditions in the laboratory for the study of brucellosis in a laminar cabinet of II protection class (Box of microbiological safety II, Laminar-S). A working two-day bacterial culture of each strain was obtained by successive three-fold passages on meatpeptone hepatic glucose-glycerol agar. Incubation was carried out for 48 hours at $37^{\circ}$ C.

After sterilization, the $\mathrm{pH}$ of the medium was 7.0-7.2. To test for sterility, each vial with the vaccine was inoculated into 2 tubes with meatpeptone agar, 2 tubes with meat-peptone liverglucose-glycerin agar and 1 bottle with meatpeptone broth. After 3-6 days of cultivation at $37-38^{\circ} \mathrm{C}$, the culture of each strain was microscoped according to the Kozlovsky method for the purity and typicality of Brucella growth.

The culture of each strain from 5 quarters washed off by the drying medium was sucked off with a siphon into sterile glass vials with a volume of 0,5 liters. In total, three experimental microseries of vaccines were prepared from strains of Brucella abortus 19UZ, 104M UZ and Brucella melitensis Rev-1 UZ in a volume of approximately $260-280 \mathrm{ml}$ each. The bacterial culture with a cryoprotectant was left in the refrigerator for 24 hours at a temperature of $2-8^{\circ} \mathrm{C}$. After 24 hours, the number of viable microorganisms was determined by the plate method. For this, a series of tenfold dilutions of each vaccine microseries was carried out. An aliquot of the suspension in a volume of $0,1 \mathrm{ml}$ was taken from each tube with the vaccine with a dilution of 1: 1000 and plated on agar nutrient medium in Petri dishes, after which the sealed dishes were placed in a thermostat for 72 hours for incubation at $37^{\circ} \mathrm{C}$. The results were recorded by counting the colonies on a daily basis. For this, the colonies grown on Petri dishes were counted, summed up, and the average value of the content of live brucella in the original tube with the cell suspension was calculated. At the same time, a White-Wilson stain was performed to determine the percentage of dissociated colonies.

\section{RESULTS AND DISCUSSIONS}

The main preliminary goal of these studies was to study the survival rate of brucella in a protective environment and the presence of the degree of dissociation.

This protective medium based on gelatin with the use of auxiliary substances, in particular sucrose, is the most studied cryoprotectant for 
bacteria. This composition was chosen based on the fact that this cryoprotectant is widely used in the production of dry live vaccines, since it is devoid of antigenic properties.

When analyzing the results obtained, it was found that in one milliliter of vaccine the number of live microbial cells varies from 180 to 190 billion microbial cells for Brucella strain abortus 104M UZ, 160-170 billion microbial cells for Brucella strain abortus $19 \mathrm{UZ}$, and for Brucella strain melitensis Rev-1 UZ - 140-150 billion microbial cells, with a significant difference in biomass yield established between the species abortus 104M UZ and melitensis Rev-1 UZ (40-50 billion microbial cells can be of significant importance in the large-scale production of vaccines.

Basic biological properties and survival of standards of industrial crops of Brucella

\begin{tabular}{|l|c|c|c|}
\hline \multirow{2}{*}{ Indicator, unit of measurement } & \multicolumn{2}{|c|}{ Indicator value for the strain } \\
\cline { 2 - 4 } & $19 \mathrm{UZ}$ & $104 \mathrm{M} \mathrm{UZ}$ & Rev $-1 \mathrm{UZ}$ \\
\hline $\begin{array}{l}\text { Accumulation of live microbes on a solid } \\
\text { nutrient medium in }\left(\mathrm{X}+\mathrm{I}_{95}\right){ }^{*} 10^{9} \text { live microbial } \\
\text { bodies / } \mathrm{ml}\end{array}$ & & & \\
\hline In test tubes & $17,0 \pm 2,0$ & $20,0 \pm 3,0$ & $13,0 \pm 3,0$ \\
\hline In mattresses & $160,0-170,0 \pm$ & $180,0-190,0 \pm$ & $140,0-150,0 \pm$ \\
& 10,0 & 15,0 & 20,0 \\
\hline Dissociation of the sample with acriflavin & Absent & Absent & Absent \\
\hline $\begin{array}{l}\text { The presence of dissociation in the thermal } \\
\text { agglutination reaction }\end{array}$ & Absent & Absent & Absent \\
\hline Dissociation level in $\left(\mathrm{X} \pm \mathrm{I}_{95}\right)$, , in percent & $1,9 \pm 3,0$ & $1,7 \pm 3,0$ & $1,3 \pm 3,0$ \\
\hline $\begin{array}{l}\text { Titer in solution with brucellosis } \\
\text { agglutinating serum antiabortus }\end{array}$ & $\begin{array}{c}\text { Not less than } \\
1000 \mathrm{IU} / \mathrm{ml}\end{array}$ & $\begin{array}{c}\text { Not less than } \\
1000 \mathrm{IU} / \mathrm{ml}\end{array}$ & $\begin{array}{c}\text { Not less than } \\
1000 \mathrm{IU} / \mathrm{ml}\end{array}$ \\
\hline
\end{tabular}

The survival rate of Brucella is quite high, which makes it possible to consider gelatin as the main cryoprotectant suitable for lyophilization of vaccine strains of Brucella.

Thus, for drying brucellosis cultures, you can use pure gelatin in a concentration of $2 \%$ with the addition of $10 \%$ sucrose, which significantly increases its efficiency. The disadvantage of a protective environment containing gelatin is the difficulty of washing away microbes from the nutrient medium due to the formation of a gel-like mass.

\section{CONCLUSIONS}

1. In the course of preliminary work, a cryoprotectant for lyophilization of brucella based on gelatin and sucrose was tested. With a given composition and an appropriate concentration of cryoprotectant, it is possible to achieve the survival of Brucella cells by freeze drying in excess of $70 \%$.

2. The disadvantage of a cryoprotectant with a gelatin content is the difficulty in washing away microbes from the nutrient medium due to the formation of a gel-like mass. 


\section{REFERENCES}

1. Kolesov S.G., Chernetsky Yu.P. Lyophilization of microorganisms and biological products. // In the book Veterinary drugs edited by D.F.Osidze 1981. p. 24-33

2. Okhapkina V.Yu., Shabalin B.A. Methods for maintaining microbial cultures. Part 1. Cryopreservation // Theoretical and Applied Ecology. 2009. № 1. P. 18-26.

3. Arkadieva Z.A. Factors affecting the viability and properties of microorganisms in different storage methods // Biological sciences. 1983. № 4. P. 93-95.

4. Timoshin A.A., Rappoport A.I., Becker M.E. Effect of drying on thermally induced structural rearrangements of the cytoplasmic membrane of yeast cells // Microbiology. 1990. V.59. Issue 2. P. 679-682.

5. Chervyakova N.S., Osin A.V. Establishing the authenticity of reference strains of pathogenic microorganisms using an automatic microbiological analyzer Vitek-2. Problems of especially dangerous infections. 2017.1.P. 100-104.

6. Pokhilenko V.D., Baranov A.M., Detushev K.V. Methods of long-term storage of collection cultures of microorganisms and development trends. Proceedings of higher educational institutions. Volga region. Medical sciences. 2009; 4 (12) p. 99-121.

7. Egorova T.A., Klunova S.M., Zhivukhina E.A. Fundamentals of Biotechnology. - 3rd edition - M .: Academy, 2006 .- 208 p.
8. Kotlov S.A. Optimization of cryoprotectants for lyophilization of SALMONELLA vaccine strains // Biological journal: electronic scientific journal. -2019 - №5(5). URL: https://bio-j.ru/archive/5/124. 